\title{
NDI of Rail Squats and Estimating Defect Size and Location Using Lock-In Thermography
}

\author{
Daren Peng, Rhys Jones \\ Department of Mechanical and Aerospace Engineering, Monash University, Melbourne, Australia \\ Email: daren.peng@monash.edu
}

Received September 24, 2012; revised October 28, 2012; accepted November 14, 2012

\begin{abstract}
Rail squats are a form of near surface rolling contact fatigue damage found in rail heads. Currently, the most popular method to detect the rail squats is utilizing ultrasonic techniques to determine their presence and measure their depths. This technique needs to be direct contact between the probe and the rail head, with a coupling fluid in at the interface. Other weaknesses of these ultrasonic techniques include false detections as well as missed detections. Infrared thermography is a relatively new non-destructive inspection technique used for a wide range of applications but is not used for rail squat detection. Lock-in thermography is a non-destructive inspection technique that can be used for the detection of near surface defects. It utilizes an infrared camera to detect the thermal waves and then produces a thermal image, which displays the local thermal wave variation in phase or amplitude. In inhomogeneous materials, the amplitude and phase of the thermal wave carries information related to both the local thermal properties and the nature of the structure being inspected. This comparison is then used to determine the phase angle difference $(\Delta \phi)$ between the input and the thermal response of the object. The aim of this paper is to determine whether lock-in thermography can be used to firstly locate squats in rails, and secondly measure their depths. It has demonstrated the feasibility for using such a technique in generating thermal responses that could be adequately utilized for the purpose of defect characterization.
\end{abstract}

Keywords: Lock-In Thermography; Phase Contrast; Infrared Camera; Squat; Non-Destructive Inspection

\section{Introduction}

Rail squats are found in rail heads all over the world due to increases in operating loads, traffic, and train speeds. They initiate at the surface and propagate down at a shallow angle of $10^{\circ}-30^{\circ}$ into the rail head to a depth of a few millimetres. After this, the crack either propagates back to the surface, causing a piece of rail to lift away (leaving a shallow cavity), or down into the rail $[1,2]$. If the first scenario occurs, which is most common, the resulting cavity causes severe vertical impact loads on rail vehicles, which can lead to further rail damage as well as damage to components of the vehicle, see Figure 1. It is therefore vital to be able to detect squats before they reach the critical stage. Therefore the development of inspection methods to assess squat presence and measure their depths are of considerable interest.

NDT methods used to detect flaws in rails included ultrasound, eddy current inspection, magnetic particle inspection, magnetic induction or magnetic flux leakage and electromagnetic acoustic transducer. Ultrasonic testing is the most common technique used to detect subsurface defects in rails [3-6]. Ultrasonic methods use high frequency sound waves which are transmitted into the rail head, and the reflected waves are measured. Their amplitudes and arrival times indicate the location and depth of defects within the rail head. Current squat detection methods in the field utilize ultrasonic techniques to firstly determine their presence and then measure their depths. This technique needs to be direct contact between the probe and the rail head, with a coupling fluid in at the interface. Other weaknesses of ultrasonic methods include false detections as well as missed detections.

NDT methods used to detect flaws in rails included ultrasound, eddy current inspection, magnetic particle inspection, magnetic induction or magnetic flux leakage and electromagnetic acoustic transducer. Ultrasonic testing is the most common technique used to detect subsurface defects in rails [3-6]. Ultrasonic methods use high frequency sound waves which are transmitted into the rail head, and the reflected waves are measured. Their amplitudes and arrival times indicate the location and depth of defects within the rail head. Current squat detection methods in the field utilize ultrasonic techniques to firstly determine their presence and then measure their depths. This technique needs to be direct contact between the probe and the rail head, with a coupling fluid in at the interface. Other weaknesses of ultrasonic methods include 


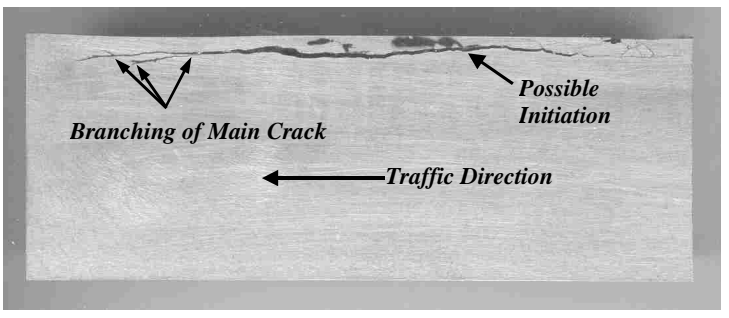

Figure 1. Subsurface cracking associated with squat defect.

false detections as well as missed detections.

Lock-in thermography utilizes a sinusoidal thermal stimulus to excite an object of interest. This stimulus can be introduced to the structure of interest internally via the thermo-elastic effect or by an external stimulus such as an array of heat lamps. When a photonic heating source is used in the lock-in technique, the technique is usually referred to as optical lock-in thermography. It can provide both amplitude and phase angle information. The phase angle refers to the measured phase difference $\Delta \phi$ between the sinusoidal input signal and the measured thermal signal response of an object. The use of lock-in thermography technique as a nondestructive evaluation technique is becoming increasingly attractive in the detection of surface or sub-surface defects in many diverse applications [7-14]. Lock-in thermography offers several advantages over other non-destructive techniques in that it is non-contact, able to inspect wide areas and produce easily interpreted results.

The aim of this paper is to evaluate lock-in thermography as a non-destructive inspection tool for rail squat defects, and determine whether it is possible to measure squat locations and depths using the technique. In the present investigation, lock-in thermography is used to detect and assess the squats in rails.

\section{Experimental Setup and Measurement Principle}

The lock-in thermography technique involves thermally exciting the surface of the specimen in a sinusoidal manner using heat lamps connected to a function generator. The thermal response of the excited surface occurs with a time delay after the oscillating heat input. The term "lock-in" refers to the need to record both input and output signals simultaneously to measure this time delay, or phase angle. A near surface defect will affect the phase angle at the surface over the defect region, thereby allowing the defect to be detected. An infra-red camera, in conjunction with a lock-in box and the appropriate software, can capture images which show the phase angle for each pixel on the image. The experimental setup for optical lock-in thermography is shown in Figure 2. A special piece of equipment known as a lock-in box is connected to the infrared camera, and is able to compare

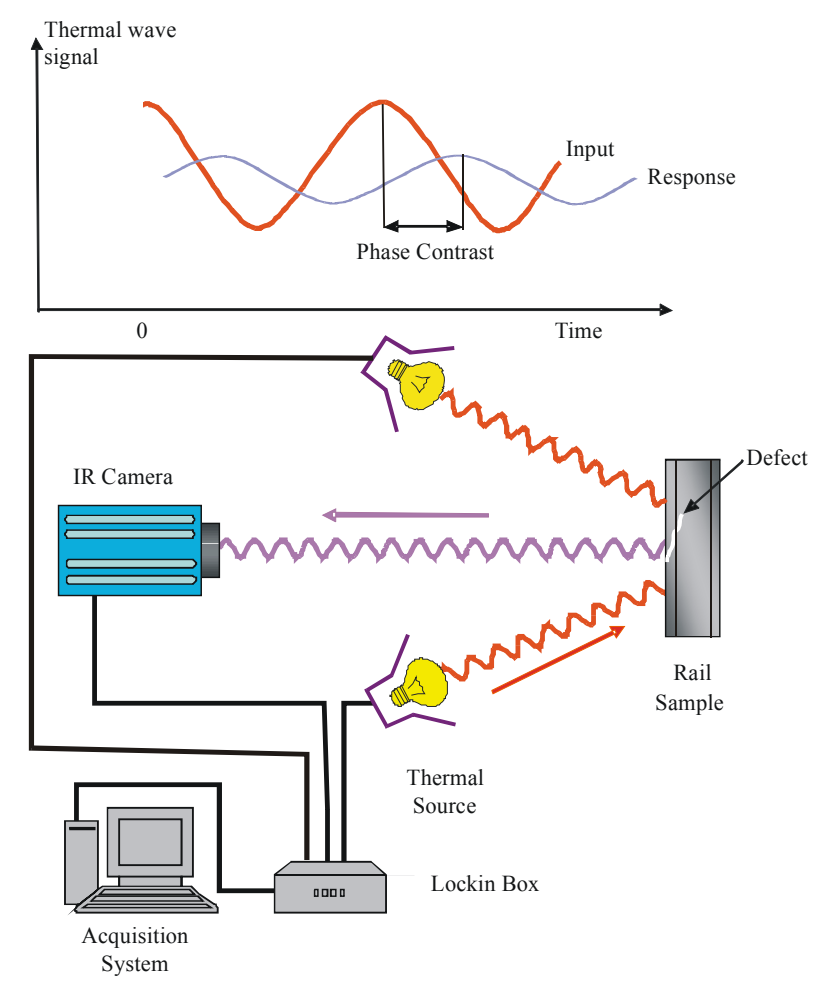

Figure 2. Schematic of experimental setup and a sinusoidal thermal input (excitation) signal with the corresponding thermal response.

the data received by the camera with the signal used to generate the periodic input.

Let's use a one dimensional mathematical model [13] describing the lockin process incorporating convective effects. The mathematical derivation is reproduced as follows: They describe the heat flux $(q)$ of the heat source with the following equation:

$$
q=\frac{Q_{o}}{2}(1+\cos (\omega t))
$$

The heat flux is composed of a constant component $\left(Q_{o} / 2\right)$ and an alterant component $\left(Q_{o} / 2\right)(\cos (w t))$. Here, $\omega$ is angular modulation frequency.

In a steady state condition the temperature distribution in a finite thickness plate of infinite dimensions can be described with the following equation.

$$
\frac{\partial^{2} T}{\partial^{2} z}-\frac{\rho C}{k} \frac{\partial T}{\partial t}=0, L \geq z \geq 0, t \geq 0
$$

Convective mechanisms occur both on the surface and the rear of the structure. Here the air is assumed to be non radiation absorbing or emitting and hence convective and radiative heat transfer components can be considered separately. Convective heat transfer $q_{c}$ can be evaluated with:

$$
q_{c}=A h_{c}\left(T_{s}-T_{\infty}\right)
$$


where $A$ is the area of the surface, $h_{c}$ is the convective heat transfer coefficient, $T_{s}$ is the temperature of the surface and $T_{\infty}$ is the temperature of the environment.

The radiative heat transfer $q_{r}$ can be written in a similar form.

$$
q_{r}=A h_{r}\left(T_{s}-T_{\text {env }}\right)
$$

where $T_{\text {env }}$ is the temperature of the radiative environment. If the body is enclosed in a large room then the temperature $T_{\text {env }}$ is equal to the temperature of the environment $T_{\infty}$. The radiative heat transfer coefficient $h_{r}$ can be defined as:

$$
h_{r}=\sigma_{S B} F\left(T_{s}^{2}+T_{\mathrm{env}}^{2}\right)\left(T_{s}+T_{\mathrm{env}}\right)
$$

where $F$ is a function of the surface radiative properties.

Thus the total heat transfer $q_{c r}$ is

$$
q_{c r}=q_{c}+q_{r}=A\left(h_{c}+h_{r}\right)\left(T_{s}-T_{\infty}\right)
$$

Using Fourier's law of heat conduction

$$
q_{z}=-k A \frac{\partial T}{\partial z}
$$

The first boundary conditions can then be developed for both the front and back surfaces of the plate.

For the front surface

$$
-k A \frac{\partial T}{\partial z}=\frac{Q_{o}}{2}(1+\cos (\omega t))-A h_{f s}\left(T_{s}-T_{\infty}\right)
$$

For the rear surface

$$
k A \frac{\partial T}{\partial z}=A h_{r s}\left(T_{s}-T_{\infty}\right)
$$

where $h_{f s}$ and $h_{r s}$ are the combined heat transfer coefficients for the front and rear surfaces respectively.

The special dependence of the $a c$ temperature component can be written in the form:

$$
T_{a c}(z)=D \mathrm{e}^{-\varsigma z}+E \mathrm{e}^{\varsigma z}, \quad \varsigma=(1+j) \sqrt{\frac{\omega}{2 \alpha}}
$$

where $D$ and $E$ are arbitrary constants and $j$ is a complex number. Thus considering the $a c$ component and applying the boundary conditions the constants $D$ and $E$ can be evaluated.

$$
\begin{aligned}
& D=\frac{Q_{o}}{2} \\
& \cdot \frac{\left(h_{r s}+k \varsigma\right) \mathrm{e}^{2 \varsigma L}}{\left(h_{f s}+k \varsigma\right)\left(h_{r s}+k \varsigma\right) \mathrm{e}^{2 \varsigma L}-\left(h_{f s}-k \varsigma\right)\left(h_{r s}-k \varsigma\right)} \\
& E=\frac{Q_{o}}{2} \\
& \cdot \frac{\left(k \varsigma-h_{r s}\right)}{\left(h_{f s}+k \varsigma\right)\left(h_{r s}+k \varsigma\right) \mathrm{e}^{2 \varsigma L}-\left(h_{f s}-k \varsigma\right)\left(h_{r s}-k \varsigma\right)}
\end{aligned}
$$

Therefore the special dependence of the $a c$ component can be written in the form:

$$
\begin{aligned}
& T_{a c}(z)=\frac{Q_{o}}{2} \\
& \cdot \frac{\left(h_{r s}+k \varsigma\right) \mathrm{e}^{(2 \varsigma L-\varsigma z)}-\left(h_{f s}-k \varsigma\right) \mathrm{e}^{\varsigma z}}{\left(h_{f s}+k \varsigma\right)\left(h_{r s}+k \varsigma\right) \mathrm{e}^{2 \varsigma L}-\left(h_{f s}-k \varsigma\right)\left(h_{r s}-k \varsigma\right)}
\end{aligned}
$$

In the case of lockin thermography measurements are usually obtained on the front surface of the body. Thus $T_{a c}$ can be written in the form:

$$
\begin{aligned}
& T_{a c}(0)=\frac{Q_{o}}{2} \\
& \cdot \frac{\left(h_{r s}+k \varsigma\right) \mathrm{e}^{2 \varsigma L}-\left(h_{f s}-k \varsigma\right)}{\left(h_{f s}+k \varsigma\right)\left(h_{r s}+k \varsigma\right) \mathrm{e}^{2 \varsigma L}-\left(h_{f s}-k \varsigma\right)\left(h_{r s}-k \varsigma\right)}
\end{aligned}
$$

The resulting phase can then be evaluated with:

$$
\phi=\operatorname{Arg}\left(T_{a c}(0)\right)
$$

\section{Thermography Experiment}

An infra-red camera (CEIP infrared systems) was placed $0.57 \mathrm{~m}$ above the surface of the rail sample. Four $750 \mathrm{~W}$ stage lights controlled by an agilent $3320 \mathrm{~A}(20 \mathrm{MHz})$ function/arbitray waveform generator provided the sinusoidal heat flux at $0.2 \mathrm{~Hz}$. Lock-in thermography works best if multiple periods are captured, as the phase angle data is effectively averaged, thus reducing noise and error from the results. This frequency $(0.2 \mathrm{~Hz})$ was used as it is low enough to detect defects up to $8 \mathrm{~mm}$ deep [15], yet high enough to allow multiple periods to be measured in a reasonable time frame. Accumulation time was 30 seconds, thus allowing six periods to be captured. Thermal images are recorded at alternate phases (In this paper, we used three locations, $I_{1}, I_{2}$ and $I_{3}$ ), over a particular thermal cycle. The pixels phase and amplitude can be calculated using the selected phase locations through the following equations:

$$
\begin{gathered}
A=\frac{2}{3} \sqrt{I_{1}^{2}+I_{2}^{2}+I_{3}^{2}-I_{1} I_{2}-I_{2} I_{3}-I_{3} I_{1}} \\
\phi=\tan ^{-1}\left[\sqrt{3} \frac{I_{1}-I_{3}}{2 I_{2}-I_{1}-I_{3}}\right]
\end{gathered}
$$

When processing the results from the experiment, the phase angle from the edge of the squat was used when finding phase contrast, as this was deemed the deepest part of the squat and was presumed to be the depth corresponding to the ultrasonic data.

Lock-in thermography tests were carried out on six rail specimens that had already been tested ultrasonically by Railcorp [16] and had their squats marked and depths recorded. The phase images produced from the lock-in 
thermography experiment successfully showed the presence of squat defects in the rail sample. All squats found by Railcorp were visible in the phase images. Squats which were missed by Railcorp's ultrasonic testing were also detected. The most prominent example of this was on Sample 5 in the region labeled "squat free". Figure 3 and Figure 4 show this region and the squats in it.

As seen in many of the images obtained, lock-in thermography is sensitive enough to detect smaller defects, such as those found in Figure 5 and Figure 6.

Ultrasonic testing of this region did not show the presence of any sub-surface defects. However, in the thermal images taken, thermal blooms characteristic of sub-surface cracking are clearly visible. Further sectioning of the rail should be able to prove the existence of sub-surface cracking in this area. These results show promise for the use of lock-in thermography to monitor the early development of squats.

Phase angle measurements for the squats were taken as an average over a small area on the phase angle image, and over a larger area for the far-field measurements. An example of the areas these measurements were taken from is shown in Figure 7. The error in the phase angle measurements was determined by taking several recordings of the same rail sample in the same position, and determining the range of measurements obtained. It

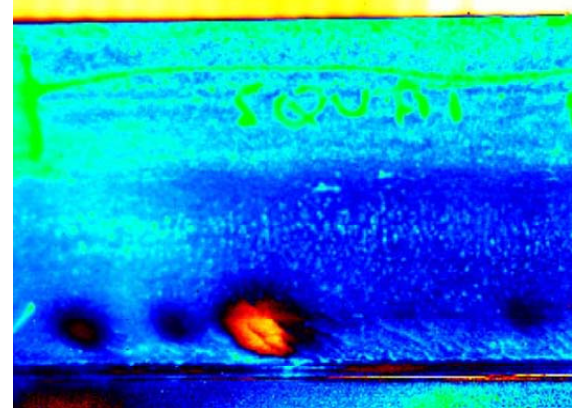

Figure 3. Phase image from left hand side of "squat free" region (by ultrasonic inspection (Sample 5)) showing one large squat and three small features which may also be squats.

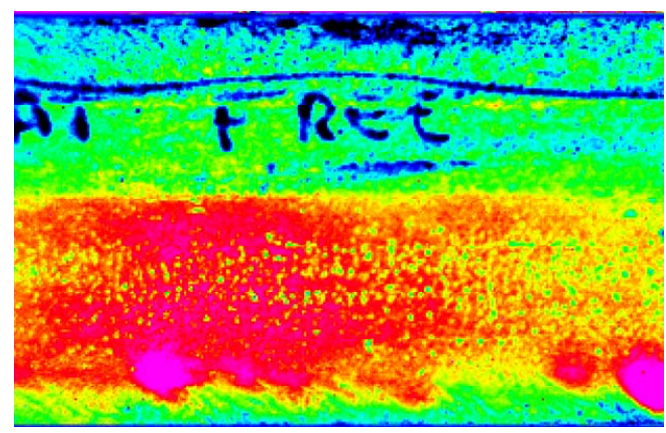

Figure 4. Phase image from right hand side of "squat free" region showing several squats.

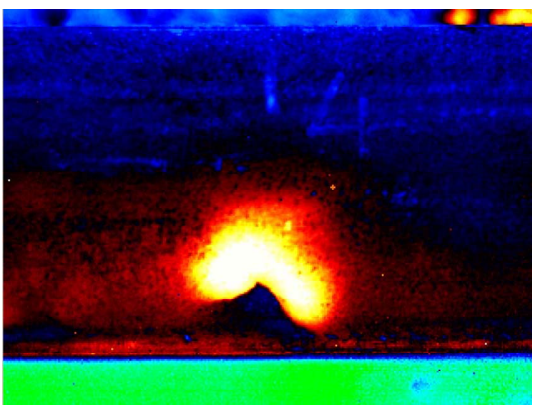

Figure 5. Phase angle image of Sample 2-mark 4.

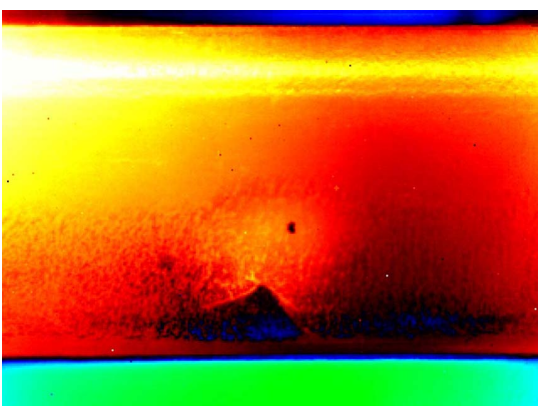

Figure 6. Temperature image of Sample 2-mark 4.

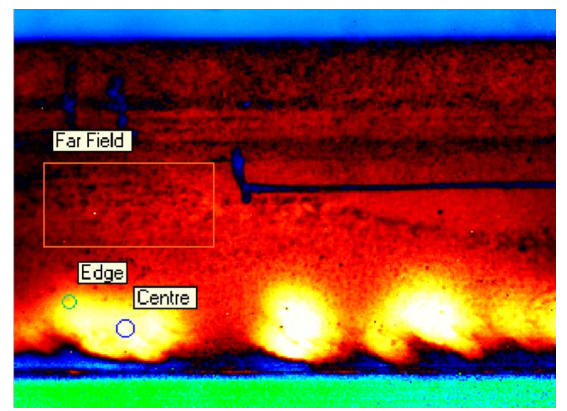

Figure 7. Example of sample used to determine phase angle measurements.

was found that the maximum range of values was approximately 1 .

\section{Calibration Technique}

The aim of "calibration study" is to explore the effects that various factors, such as defect size and crack profile shape, have on the thermal response. It can be performed by extensive experimental pre-tests or numerical modeling with necessity for (artificial) representations of a defect. For squats, which are complex in shape and of unknown size, the "calibration study" with experimental method will lead prediction redundant. The research outlined in this paper examines the use of $3 \mathrm{D}$ finite element modeling (FEM) as a potential flexible tool in generating a "calibration curve" that would relate phase contrast to squat depth so that anyone who conducted a lock-in thermography experiment on rail squats could determine 
their depths based on the phase images.

The Finite Element Analysis was handled by NEiNastran [17] in the transient heat analysis mode. A 0 - 30 $\mathrm{W} / \mathrm{m}^{2}$ sinusoidal heat flux was applied to the top surface of all models at $0.2 \mathrm{~Hz}$. The heat input function defined in FEMAP [18] was at $0.1 \mathrm{~s}$ intervals for 200 time steps. Likewise the FEA was conducted in NEi-Nastran for 200 time steps at $0.1 \mathrm{~s}$ intervals, thus generating temperaturetime histories of each node for 20 seconds. The initial temperature of each model was set to $293 \mathrm{~K}$ to approximate the temperature in the lab where the experiments were conducted. The material thermal properties used in this paper were: specific heat $-490 \mathrm{~J} / \mathrm{kg} \cdot \mathrm{K}$, coefficient of Thermal expansion-14 $\times 10^{-5}$, thermal conductivity$47.5 \mathrm{~W} / \mathrm{mK}$ and free air convection heat transfer coefficient $-25 \times 10^{-6} \mathrm{~W} / \mathrm{K} \cdot \mathrm{m}^{2}$. The density of material is set at $7870 \mathrm{~kg} / \mathrm{m}^{3}$ and Stefan-Bolzmann constant is $56.7 \times$ $10^{-9} \mathrm{~W} / \mathrm{m}^{2} \mathrm{~K}^{4}$.

To investigate the independent effects of depth on phase contrast we performed a 3-dimensional thermal FEA. A round flat bottom hole model was constructed at a constant size of $20 \mathrm{~mm}$ with depths ranging $0.5-6 \mathrm{~mm}$, see Figure 8. The depth range was chosen as it covered the depth data provided by Railcorp as well the typical squat depths quoted in the literature. To investigate the effect of size, several 3D finite element models were constructed with sizes of $25 \mathrm{~mm}, 20 \mathrm{~mm}, 14 \mathrm{~mm}$ and 8 $\mathrm{mm}$. The phase contrast measured over the defects as a function of defect depth for a thermal excitation frequency of $0.2 \mathrm{~Hz}$ was shown in the Figure 9. There is an exponential decay in phase contrast as depth increases and there are no observed different in phase contrast for the defects with the diameter of $20 \mathrm{~mm}$ and $25 \mathrm{~mm}$. Therefore, the phase contrast "calibration" of the defect depth results generated the $3 \mathrm{D}$ finite element model with defect diameter of $20 \mathrm{~mm}$ can be used to predict depth of squat in rail. It should be noted that the phase contrast at the edge of the defect is approximately half of that seen in the middle. This has critical implications on real lock-in thermography experiments as it shows that the phase contrast cannot simply be measured over the middle of the squat as was done for the thermography experiment conducted in this paper. Instead, the phase contrast from the edge should be used, assuming it is the deepest point of the squat.

To examine the effect of altering the heat loads, a heat flux with peak magnitude of $30 \mathrm{~W} / \mathrm{m}^{2}$ was used for all finite element models and this peak magnitude was halved to $15 \mathrm{~W} / \mathrm{m}^{2}$ for one analysis. The brief investigation on heat load effects showed that phase contrasts were unaffected by halving the amplitude of the input flux. This is a significant finding as it means lock-in thermography could potentially be used in the field, and the phase images produced would not be affected by dif-

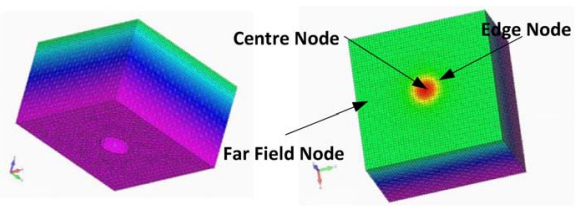

Figure 8. The model and temperature distribution a for 3D flat bottom hole model with $0.2 \mathrm{~Hz}$ excitation rate and 10 Hz sampling rate.

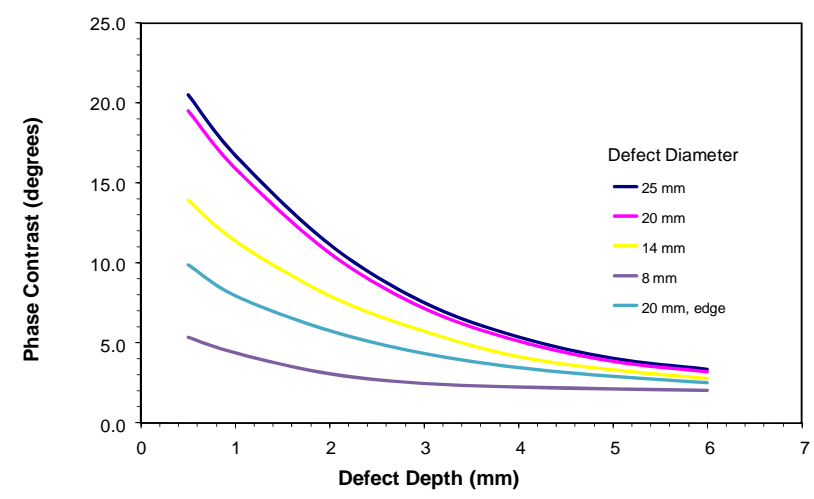

Figure 9. The phase contrast for various sampling rate obtained with a thermal excitation frequency of $0.2 \mathrm{~Hz}$.

fering heat inputs or convective losses, for example if different heat lamps were used or if a breeze was present. Now, the phase contrast "calibration" of the defect depth results generated by the $3 \mathrm{D}$ finite element model can be used to predict depth of squat in rail from lock-in thermography experimental phase images.

\section{Squat Examination}

The aim of this investigation was to verify the squat depths ultrasonic measure supplied by Railcorp. Sample 6 was chosen to be cut into segments as it gave the clearest phase images from the thermography experiments. Sample 6 contained six squat markings (1, 2, 3, 4, 5 and 6), however Squat 2 was not cut as it was considered too close to Squat 1. The phase image at location 5 showed that two clear squats exist. As such, the squats marked for cutting were numbers $1,3,4,5 \mathrm{a}, 5 \mathrm{~b}$ and 6 . The resulting segments were labeled A to G. Figure 10 shows the relationship between squats and segments.

Digital images of each crack surface were captured with a scale visible. Since each thermography image also has a corresponding scale image it was possible to locate the precise location of a squat edge on its corresponding phase image. The phase contrast at that precise point was then recorded for each squat and plotted against its depth, as measured physically. Also, the phase contrasts were graphed along the length of the cut for these squats, see Figures 11-22. Table 1 outlines the results of the depth relative to surface measured during the squat examination, ultrasonic test and lock-in thermography experiment. 


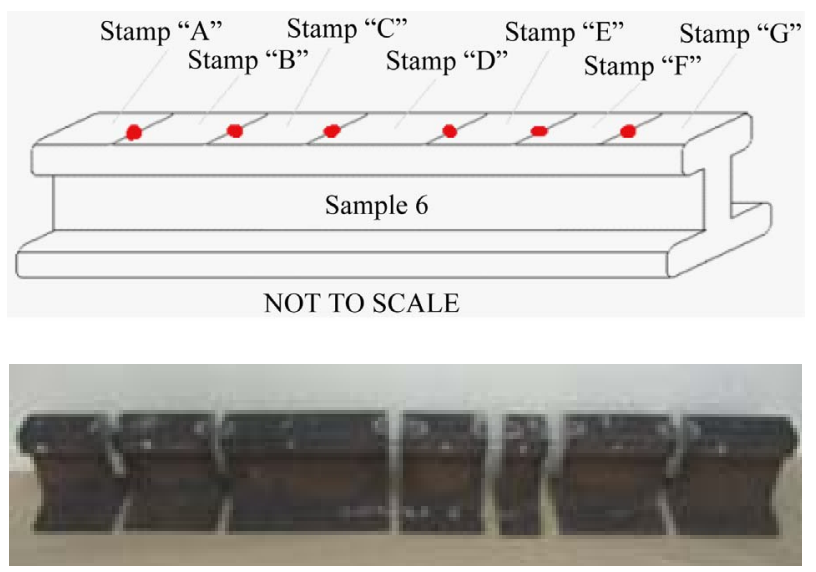

Figure 10. Diagram showing segment labeling convention for Sample 6, which was segmented.

The percentage difference between measured crack depths and Railcorp's ultrasonically measured depths as well as the measured depth compared to lock-in thermography measured depth has been provided in this table.

It was found that the actually angles of propagation were shown to be approximately $16^{\circ}-20^{\circ}$, in line with the literature stating the squat cracks generally propagate down at $10^{\circ}-30^{\circ}$ relative to the surface. In addition, it can be seen that the phase contrast at the edge (deepest point of the crack) is approximately $7.6 \%-16 \%$ of the experimental measured for each squat. More squats need to be cut open and examined before any firm conclusion can be drawn from this, but it may be possible to approximate the location of the edge of a squat by examining its phase contrast as a function of the distance from surface crack. It should be noted that the there was a large discrepancy between the depths measured ultrasonically and the depths measured physically. Of course, the depths measured during the squat examination are only the depths of the crack along the cutting plane, so there may be deeper parts of the squat. Table 1 shows the difference in crack depth relative to the ultrasonic data varies from approximately $-36 \%$ to $+61 \%$.

The experiments showed that there did seem to be a very vague relationship between phase contrast and squat depth, however it was difficult to judge where the deepest point of the squat is based on the phase images alone, so there was a high degree of uncertainty in relation to those particular results. It was found by cutting up Sample 6 that the data provided by Railcorp contains up to $61 \%$ error in depth (for Sample 6 at least). This highlights the need to more carefully determine actual squat depths and the locations of maximum depth before trying to determine the relationship with phase contrast. For the majority of the depth of the squat, there was a reasonable good correlation between the true squat depth and the numerical estimates. However, it was also found that for deeper defects (i.e. greater than $5 \mathrm{~mm}$ ) the estimated and true

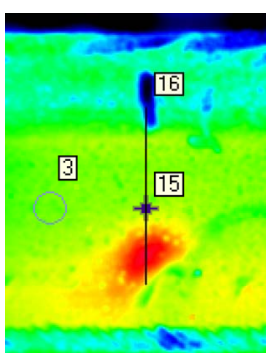

(a)

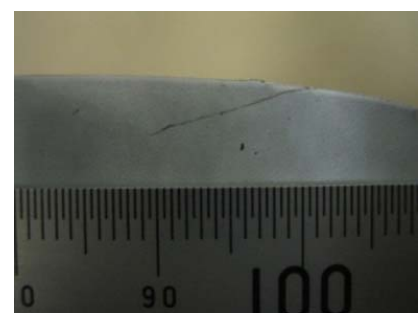

(b)
Figure 11. (a) Location of cut (16), location of actual squat edge (15), far field reference point (3); (b) Squat 1 shown on segment $B$. Depth $=4.93 \mathrm{~mm}$, size $=15.28 \mathrm{~mm}$, angle from surface $=18.18^{\circ}$.

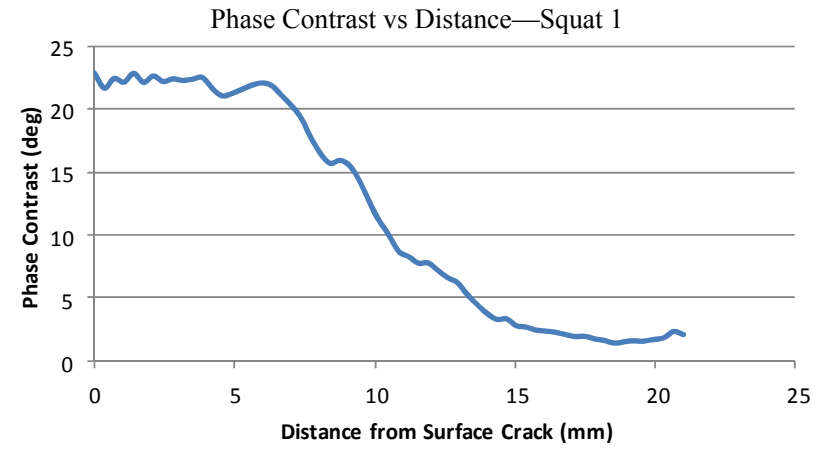

Figure 12. Phase contrast versus distance from surface crack for Squat 1.

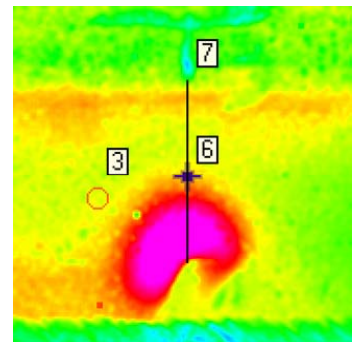

(a)

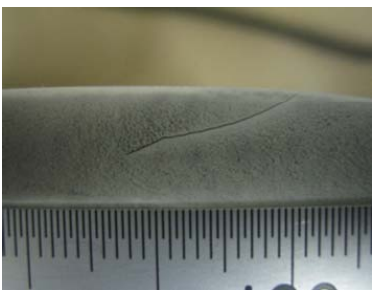

(b)
Figure 13. (a) Location of cut (7), location of actual squat edge (6), far field reference point (3); (b) Squat 3 shown on segment $C$. Depth $=5.33 \mathrm{~mm}$, size $=14.89 \mathrm{~mm}$, angle from surface $=20.13^{\circ}$.

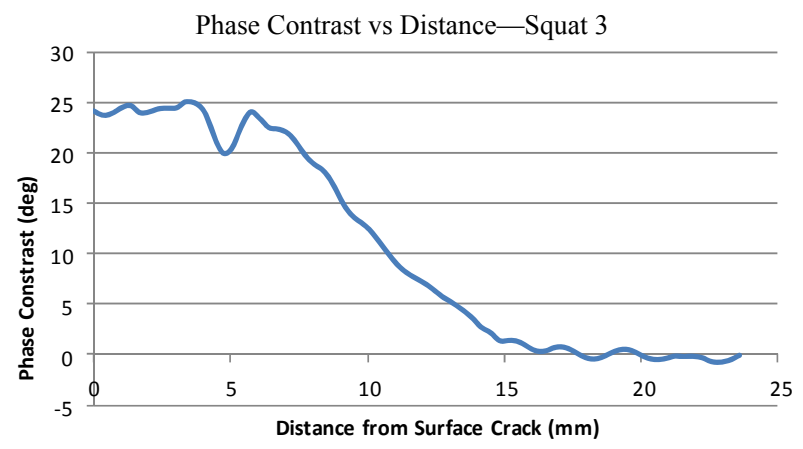

Figure 14. Phase contrast versus distance from surface crack for Squat 3. 


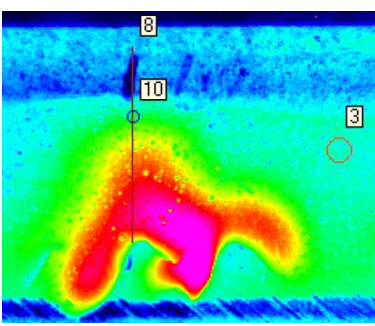

(a)

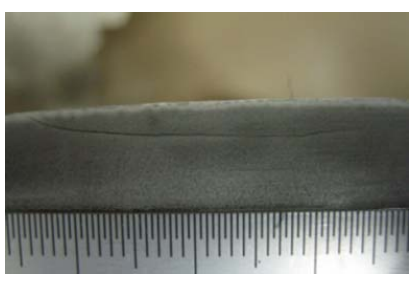

(b)
Figure 15. (a) Location of cut (8), location of actual squat edge (10), far field reference point (3); (b) Squat 4 shown on segment C. Depth $=3.07 \mathrm{~mm}$, size $=27.13 \mathrm{~mm}$.

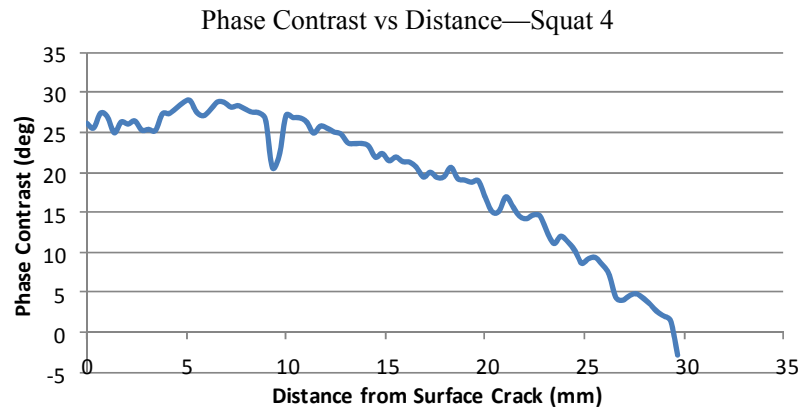

Figure 16. Phase contrast versus distance from surface crack for Squat 4.

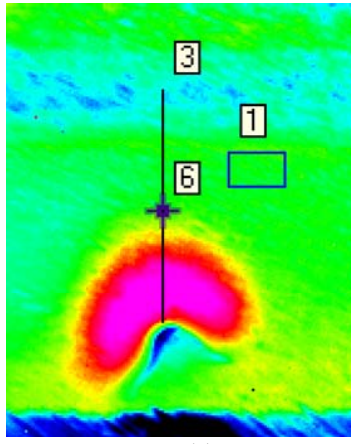

(a)

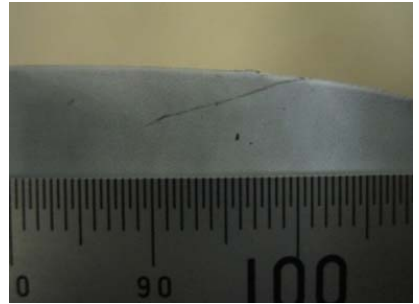

(b)
Figure 17. (a) Location of cut (3), location of actual squat edge (6), far field reference point (1); (b) Squat 5a shown on segment $E$. Depth $=4.91 \mathrm{~mm}$, size $=15.87 \mathrm{~mm}$, angle from surface $=17.36^{\circ}$.

Phase Contrast vs Distance-_Squat 5a

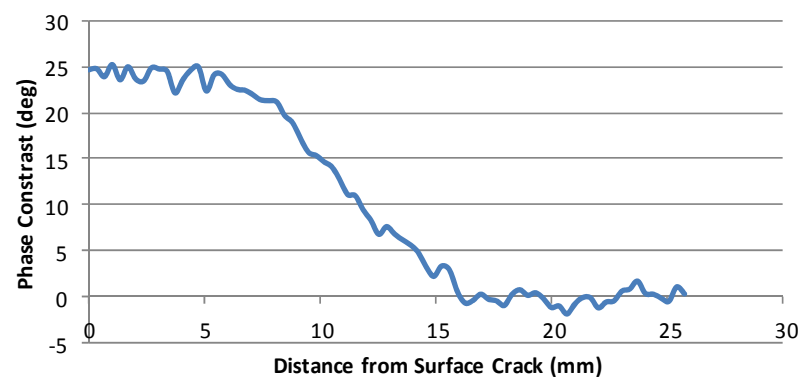

Figure 18. Phase contrast versus distance from surface crack for Squat 5 a.

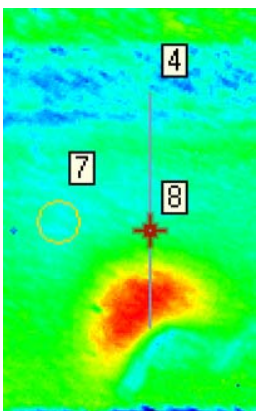

(a)

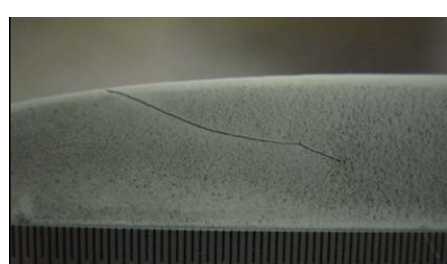

(b)
Figure 19. (a) Location of cut (4), location of actual squat edge (8), far field reference point (7); (b) Squat $5 b$ shown on segment E. Depth $=5.37 \mathrm{~mm}$, size $=\mathbf{1 3 . 8 4} \mathrm{mm}$, angle from surface $=21.80^{\circ}$.

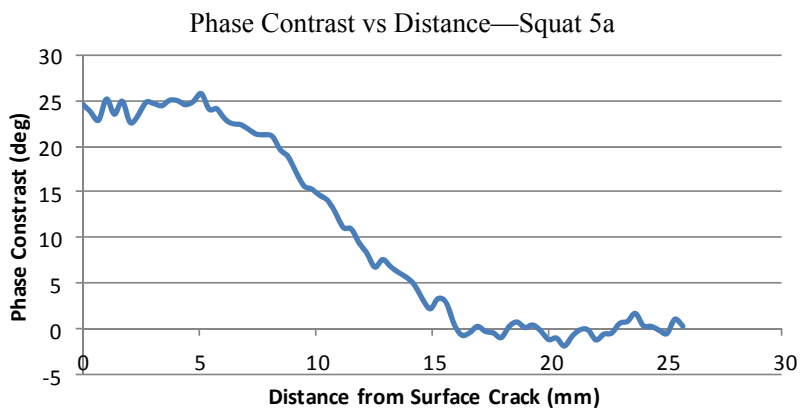

Figure 20. Phase contrast versus distance from surface crack for Squat $5 b$.

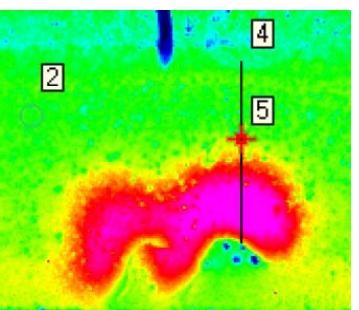

(a)

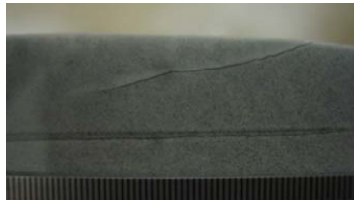

(b)
Figure 21. (a) Location of cut (4), location of actual squat edge (5), far field reference point (2); (b) Squat 6 shown on segment G. Depth $=4.92 \mathrm{~mm}$, size $=\mathbf{1 6 . 8 3} \mathrm{mm}$, angle from surface $=16.50^{\circ}$.

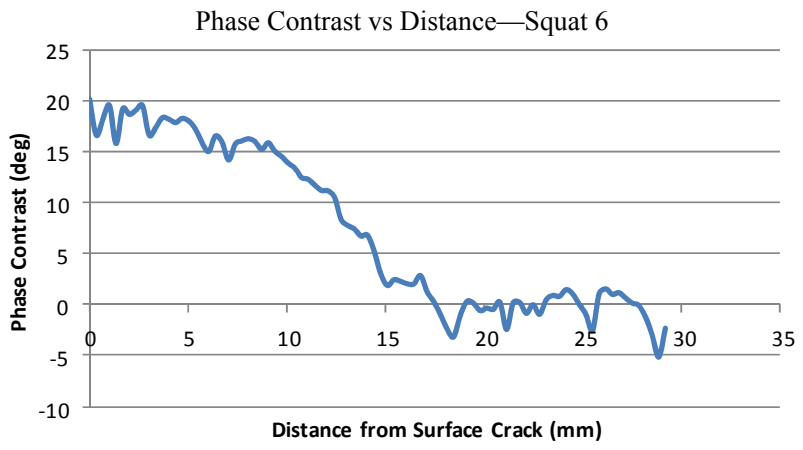

Figure 22. Phase contrast versus distance from surface crack for Squat 6. 
Table 1. Summary of squat dimensions found by cutting Sample 6 into segments.

\begin{tabular}{cccccc}
\hline \multirow{2}{*}{ Squat } & $\begin{array}{c}\text { Cross-section } \\
\text { examination }\end{array}$ & $\begin{array}{c}\text { Ultrasonic } \\
\text { inspection }\end{array}$ & $\begin{array}{c}\text { Depth } \\
\text { diff (\%) }\end{array}$ & $\begin{array}{c}\text { Lock-in } \\
\text { thermography }\end{array}$ & $\begin{array}{c}\text { Depth } \\
\text { diff (\%) }\end{array}$ \\
\cline { 2 - 5 } Depth (mm) & Depth (mm) & & Depth (mm) & \\
\hline 1 & 4.93 & 3.3 & 49.4 & 5.55 & 12.5 \\
3 & 5.33 & 3.3 & 61.6 & 4.62 & -13.3 \\
4 & 3.07 & 4.8 & -36 & 3.30 & 7.6 \\
$5 \mathrm{a}$ & 4.91 & 4 & 22.8 & 5.46 & 11.2 \\
$5 \mathrm{~b}$ & 5.37 & 4 & 34.3 & 6.23 & 16.0 \\
6 & 4.92 & 4.6 & 7 & 5.34 & 8.5 \\
\hline
\end{tabular}

squat depth differed by approximately $12 \%-16 \%$.

In addition, a rule of thumb for lock-in thermography is that only a defect whose diameter is greater than its depth can be detected, as mentioned in [15]. This should not be a problem in the context of this project, however, as rail squats are generally less than $6 \mathrm{~mm}$ deep, while their size (looking from above) is substantially larger than this.

The experiments showed that lock-in thermography is capable of revealing squats of various sizes and depths. The phase images produced also showed the presents of squats that were missed by the ultrasonic testing. This is a significant finding as it demonstrates the major strength of thermography over other NDI techniques: the ability to cover a wide area quickly, simultaneously, and without contact. Interestingly, it was found that altering the amplitude of the sinusoidal heat input and introducing large convective losses did not alter the phase response at the surface of the material. This is a useful finding as it means only material properties and defect geometry will alter the phase images from further experiments.

\section{Conclusions}

A brief summary of the findings of the aforementioned investigations are presented as follow. Non-destructive inspection of the six rail specimens with squats had been tested by lock-in thermography technique. A 3-dimensional thermal finite element model was used to investigate the independent effects of depth on phase contrast. One rail sample was cut into sections to measure the depth of each squat.

Overall this approach found that lock-in thermography is a useful NDI tool to determine the presence and location of rail squats. However at this stage, it is difficult to measure their depths accurately based on phase images. The condition of rough top surface of track has effect on the phase contrast image obtained through lock-in thermogrphy. Another variable which was unable to be quantified in the experimental data is the possibility of foreign material within the crack, such as water, lubricant, or even the presence of rust on the crack face. These vari- ables could potentially affect the accuracy of the phase angle measurements, and further research is needed to determine the effects they may have.

\section{Acknowledgements}

The authors would like to acknowledge the assistance of Dr. M. Krishnapillai and Mr. M. Plozza.

\section{REFERENCES}

[1] M. Kerr, A. Wilson and S. Marich, "The Epidemiology of Squats and Related Rail Defects," Proceedings of CORE 2008 Conference on Railway Engineering: Rail; The Core of Integrated Transport, Perth, 2008, pp. 83-96.

[2] S. Marichand S. Mackie, "The Development of Squat Defects under High Axle Load Operations," CORE 2002: Cost Efficient Railways through Engineering, Wollongong, 2002, pp. 17-26.

[3] G. Zumpano and M. Meo, "A New Damage Detection Technique Based on Wave Propagation for Rails," International Journal of Solids and Structures, Vol. 43, No. 5, 2005, pp. 1023-1047.

[4] P. C. Johnson and S. Wise, "Significance of a Detection of Defects in Rails," Non-Destructive Testing, Vol. 3, No. 2, 1970, pp. 111-116. doi:10.1016/0029-1021(70)90100-3

[5] D. E. Bray and M. Najm, "Ultrasonic Angle-Beam Inspection through the Cold-Worked Layer in Railroad Rail," NDT \& E International, Vol. 18, No. 3, 1985, pp. 139-145. doi:10.1016/0308-9126(85)90198-1

[6] R. Clark, "Rail Flaw Detection: Overview and Needs for Future Developments," NDT \& E International, Vol. 37, No. 2, 2004, pp. 111-119. doi:10.1016/j.ndteint.2003.06.002

[7] D. Wu, G. Zenzinger, W. Karpen and G. Busse, "Nondestructive Inspection of Turbine Blades with Lock-In Thermography," Materials Science Forum, Vol. 210-213, 1996, pp. 289-295.

doi:10.4028/www.scientific.net/MSF.210-213.289

[8] C. Meola, R. D. Maio, N. Roberti and G. M. Carlomagno, "Application of Infrared Thermography and Geophysical Methods for Defect Detection in Architectural Structures," Engineering Failure Analysis, Vol. 12, No. 6, 2005, pp. 875-893. doi:10.1016/j.engfailanal.2004.12.030

[9] C. Meola, G. M. Carlomagno, A. Squillace and A. Vitiello, "Non-Destructive Evaluation of Aerospace Materials with Lock-In Thermography," Measurement Science and Technology, Vol. 13, No. 3, 2006, pp. 380-389.

[10] D. Wu, A. Salerno, U. Malter, R. Aoki, R. Kochendorfer, P. K. Kachele, K. Woithe, K. Pfister and G. Busse, "Inspection of Aircraft Structural Components Using LockIn-Thermgraphy," Quantitative Infrared Thermography, QIRT 96, Edizione ETS, Pisa, 1997.

[11] C. Meola, G. M. Carlomagno, A. Squillace and G. Giorleo, "Non-Destructive Control of Industrial Materials by Means of Lock-In Thermography," Measurement Science and Technology, Vol. 13, No. 10, 2002, pp. 1583-1590. doi:10.1088/0957-0233/13/10/311 
[12] A. Dillenz, T. Zweschper, G. Riegert and G. Busse, "Progress in Phase Angle Thermography," Review of Scientific Instruments, Vol. 74, No. 1, 2003, pp. 417-421. doi: $10.1063 / 1.1524010$

[13] W. Bai and B. S. Wong, "Photothermal Models for LockIn Thermographic Evaluation of Plates with Finite Thickness under Convection Conditions," Journal of Applied Physics, Vol. 89, No. 6, 2001, pp. 3275-3283. doi:10.1063/1.1344916

[14] G. Busse, D. Wu and W. Karpen, "Thermal Wave Imaging with Phase Sensitive Modulated Thermography," Journal of Applied Physics, Vol. 71, No. 8, 1992, pp. 39623966. doi:10.1063/1.351366
[15] C. D. Wallbrink, S. Wade and R. Jones, "The Effect of Size on the Quantitative Estimation of Defect Depth in Steel Structures Using Lock-In Thermography," Journal of Applied Physics, Vol. 101, No. 10, 2007, Article ID: 104907. doi:10.1063/1.2732443

[16] M. Kerr and A. Wilson, "Railcorp Squat Samples," Railcorp Report, Australia, 2008.

[17] NEiNastran User's Manual, Version 10, Noran Engineering, Inc., California, 2010.

[18] FEMAP, "Finite Element Modelling and Post Processing ${ }^{\mathcal{C}}$, Version 10.0.2, Structural Dynamics Research Corporation, Pennsylvania, 2009. 


\section{Appendix}

The image recording is synchronized with the modulation frequency. The IR camera takes three images within one cycle (Figure A, T is the period of a cycle).The lock-in system obtains three signal values $I_{1}, I_{2}$ and $I_{3}$ in every pixel of the image. Hence, for every wave cycle, each image pixel provides three times data, which differ in phase by $120^{\circ}$. With these three data, the local magnitude $(A)$ and phase $(\phi)$ of the temperature modulation can be found.

Hence, for every wave cycle, each image pixel provides three times data, which differ in phase by $120^{\circ}$.

$$
\begin{gathered}
I_{1}=I_{0}+I_{a} \cos \phi \\
I_{2}=I_{0}+I_{a} \cos (\phi+\alpha) \\
I_{3}=I_{0}+I_{a} \cos (\phi+2 \alpha)
\end{gathered}
$$

where $I_{0}$ is the average intensity, $I_{a}$ is the intensity modulation, $\phi$ is the phase, and $\alpha$ is the phase step size. Even though $\alpha$ can be any value. The fast phase-shifting algorithm described in this paper $\alpha$ is $2 \pi / 3$. For $\alpha=120^{\circ}$, solving Equations (A1) to (A3) for the phase and local magnitude yields

$$
\phi=\tan ^{-1}\left[\sqrt{3} \frac{I_{1}-I_{3}}{2 I_{2}-I_{1}-I_{3}}\right]
$$

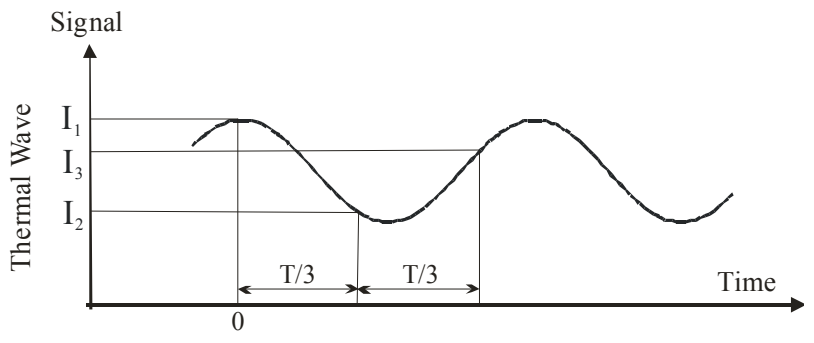

Figure A. Thermal wave response relative to a lock-in signal with three signal values $I_{1}$ to $I_{3}$ at a pixel.

$$
A=\frac{2}{3} \sqrt{I_{1}^{2}+I_{2}^{2}+I_{3}^{2}-I_{1} I_{2}-I_{2} I_{3}-I_{3} I_{1}}
$$

In actuality, the system takes more than just four pictures $I_{1}, I_{2}$ and $I_{3}$ for the calculation of its phase angle and magnitude. An average of more than three images percycle over several modulating cycles is then taken. However, due to the sinusoidal modulation, all the images are eventually consolidated into only three basic images $\left(I_{1}\right.$, $I_{2}$ and $I_{3}$ ). The phase angle and magnitude values of these images are obtained from Equations (16) and (17). 